\title{
Managing cannabis use in people with severe mental illness: what can be done ${ }^{\dagger}$
}

\section{Zerrin Atakan}

\begin{abstract}
SUMMARY
Nearly half of people with severe mental illness use cannabis sometime in their lives and during their illness. Its use can have multiple and severe consequences for the course of the illness. Despite the significance of the problem, managing cannabis use in this group is a recently developing topic and is still in its infancy. This article reviews the current state of knowledge on the management of people with severe mental illness who continue to use cannabis, specifically focusing on different models of service provision, and psychological and pharmacological interventions.
\end{abstract}

\section{DECLARATION OF INTEREST}

None.

Over the past decade, a number of treatment models have been proposed for people with severe mental illness and substance use problems. One of these initiatives, originating in the USA but now taken up in the UK, was to create specifically designed services to tackle this dual diagnosis. In both countries, addiction and generic mental health services have historically functioned separately and have considerable differences in terms of culture, philosophy and treatment strategies. For instance, generic services prefer more assertive approaches to maintain patients in treatment, whereas addiction services encourage individuals to take responsibility in their own treatment. Major cultural shifts are not easy to implement and maintain within services. One of the aims in creating specific dual diagnosis services was to overcome some of these difficulties.

Thus far, three service models have been developed: serial treatment, in which the treatment of psychosis is completed before substance use treatment begins; parallel treatment, in which both problems are treated at the same time, but by different teams; and integrated treatment, in which the same team treats both problems simultaneously. The integrated treatment model has particular benefits for the patient, but it requires well-integrated in-patient care, staff trained to deal with both mental health and substance use problems, assertive community services and supportive living environments. This model can be costly and requires either better use of existing resources or the creation of new ones. All service delivery models aim to provide motivation-based treatments as well as psychosocial methods.

\section{How successful are these models?}

It seems only sensible that these treatment models should be fully developed and tested before widespread implementation (Weaver 1999). However, dual diagnosis services have been set up in the USA and the UK on the basis of models that lack clear evidence even of superiority over standard care. A systematic Cochrane review (Cleary 2008) to evaluate the efficacy of treatment programmes for people with both severe mental illness and substance misuse identified 25 randomised controlled trials and found no compelling evidence to support any one psychosocial treatment over another in the reduction of substance use or improvement of mental state. It was concluded that existing methodological difficulties hindered pooling and interpreting results because of high drop-out rates, varying outcome measures and comparison groups. The same review, however, concluded that individual studies provided some support in favour of motivational interviewing for substance use reduction and that this intervention is a crucial component in cognitive-behavioural therapy for substance use.

One study mentioned in the Cochrane review, however, reported that a residential programme had a significantly greater chance of retaining people in care than a non-residential approach (Burnam 1995).

Despite the lack of unequivocal evidence for an efficient model for managing substance use in severe mental illness, motivational interviewing techniques, community reinforcement approaches, assertive case management, and a blend of standard psychological interventions for substance use with specific pharmacotherapies have each proven successful in extending abstinence (Ziedonis 1997; Brunette 2006a).

This section is summarised in Key points 1 .

\section{Psychological interventions}

People with severe mental illness are often denied psychological interventions, even if they ask for
Zerrin Atakan worked as Lead Consultant in the National Psychosis Unit at the Maudsley \& Bethlem Royal Hospitals in London until August 2007, when she retired from National Health Service duties. She currently holds an honorary contract at the Institute of Psychiatry in London, where she is doing research on the effects of cannabis compounds on brain functioning and development of an intervention model for cannabis-using patients with psychosis. As a consultant psychiatrist she gained extensive expertise treating severe mental illness. She has advised the Home Office, voluntary mental health organisations and local councils on cannabis use, and written for cannabis websites to inform young people about the effects of the drug on mental health.

Correspondence Dr Zerrin Atakan, Institute of Psychiatry, De Crespigny Park, London SE5 8AF, UK. Email: Zerrin. Atakan@iop.kcl.ac.uk

This is the second of two articles by Zerrin Atakan in Advances on cannabis use in people with severe mental illness. The first, which addressed the extent of the problem, its assessment and the reasons why cannabis is used, appeared in the previous issue of the journal (2008; 14: 423-31।. 
KEY POINTS 1 Three service models for people with dual diagnosis

\begin{tabular}{|ll|}
\hline $\begin{array}{l}\text { - Serial treatment: one treatment is followed } \\
\text { by the other }\end{array}$ & $\begin{array}{l}\text { - Integrated treatment requires: } \\
\text { well-integrated in-patient and substance }\end{array}$ \\
- Parallel treatment: concurrent but separate & misuse treatments and assertive community \\
treatments are delivered by two teams & services \\
- Integrated treatment: both treatments are & assertive styles of engagement \\
delivered by the same team & supportive living environments \\
- Serial and parallel treatment programmes & - There is no clear evidence that any one of \\
require: & the three models is better than the others or \\
seamless planning & than standard care \\
time management & \\
special care not to overload the patient &
\end{tabular}

FFor a discussion of this approach see Treasure J (2004) Motivational interviewing. Advances in Psychiatric Treatment; 10: 331-7. Ed. them. This is due partly to lack of resources and partly to the belief that they cannot benefit from such treatment, because of their illness and cognitive deficits. Interestingly, a study of potential participants in the Clinical Antipsychotic Trials of Intervention Effectiveness (CATIE) programme found that the negative symptoms of schizophrenia had very little effect and positive symptoms had no effect on decision-making capacity (Stroup 2005). The majority of people with schizophrenia can potentially benefit from psychological interventions and participate in shared decisionmaking. People who have bipolar disorder show even better cognitive performance than those with schizophrenia (Krabbendam 2005).

\section{Motivational interviewing}

Over the past two decades several psychological intervention methods have been developed to provide patient-tailored treatment programmes for tackling substance misuse, and some of these have also been used with people with severe mental illness. These methods adapt treatment to the motivational level of the individual. Motivational interviewing therapies ${ }^{\ddagger}$ can be based on a five-stage scale which indicates the individual's readiness to change (Box 1).

The aim of motivational interviewing and motivational enhancement therapy for substance misuse is to strengthen the patient's motivation to change by increasing their awareness of the impact of substance use and encouraging them to take responsibility for their behaviour (Miller 2002). The therapist is empathetic, open, non-judgemental and realistic about what can be achieved. Giving advice, reassurance or immediate problem-solving are usually avoided. The interview is strategically directed towards the patient's use of substances and related life events. For some patients the goal of therapy will be harm reduction, for others it will be cessation or maintenance.
The efficacy of motivational interviewing for primary substance use problems has been well established, and several studies have examined its use to treat substance misuse in people with severe mental illness. It seems that some tailoring of the approach is required for people with psychotic disorders. In a large population of (non-psychotic) university students who had been using substances, 3-month follow-up revealed that a single session of motivational interviewing had reduced their use of cigarettes, alcohol and cannabis, albeit mainly through moderation rather than cessation (McCambridge 2004). However, studies indicate that a single standard session would not be sufficient for people with severe mental illness. One such study, involving individuals with psychotic disorders and co-occurring substance use problems, recommends modifications to standard motivational interviewing to take into account patients' cognitive deficits by simplifying openended questions, refining reflective thinking skills, heightening emphasis on affirmations and integrating psychiatric issues into personalised feedback (Martino 2002). It stresses theimportance of considering the patients' clinical state and the events they are experiencing during treatment, as these may need to be tackled in the therapy sessions. As in standard motivational interviewing, there is emphasis on reflective listening, discussing obstacles to treatment and identifying factors that would improve motivation.

\section{Combination therapies}

To deal with the 'dual' set of problems presented, some psychosocial treatment programmes for co-occurring psychotic disorders and substance misuse combine various therapy models. For instance, for an individual in the action stage of change (Box 1), relapse prevention may involve a hybrid behavioural therapy that integrates substance misuse treatment, relapse prevention and

BOX 1 The five stages of change in relation to substance misuse

1 Pre-contemplation: continuous use with no interest in quitting in past 6 months

2 Contemplation: continuous use with ambivalent interest in quitting

3 Preparation: continuous use with interest in quitting in the next 30 days

4 Action: active attempt to quit

5 Maintenance: abstinent for more than 3 months but less than 5 years

(After Prochaska 1992) 
social skills training. It is expected that the needs of the patient will be different at each stage. For an individual moving from the action stage to the maintenance stage, special attention may be given, for example, to restoring relationships, seeking employment or engaging in meaningful activities in social settings that include non-users.

\section{Motivational enhancement plus cognitive-behavioural therapy}

Various combination therapies for out-patients have been subjected to trials. One example is a manualised group-based intervention for reducing substance use (James 2004). Based on the principles of motivational enhancement and cognitivebehavioural therapy, the 6-week intervention involved weekly 90-min sessions tailored to the patient's stage of change and motivational level. In this Australian randomised controlled study, 63 participants were allocated to the intervention or to a single educational session. Of these, 92\% completed a 3-month follow-up assessment of psychopathology, antipsychotic dose, alcohol and substance use, severity of dependence and hospitalisation. Significant improvements were found on most outcome measures in the treatment group in comparison with the control group. However, a limitation of the study is the short period to follow-up.

A UK-based randomised controlled study evaluated the efficacy of individual and family-oriented cognitive-behavioural therapy for treatmentresistant psychosis, combined with motivational interviewing for substance use problems; the treatment group received about 29 sessions of combined therapy (Barrowclough 2001). Significant improvements in functioning were found in the treatment group at 18-month follow-up (Haddock 2003). The cost was similar to that of the control treatment.

\section{Insight-adherence-abstinence in first-episode schizophrenia}

A therapy developed specifically for patients with first-episode schizophrenia who are using cannabis focuses on the triad 'insight-adherenceabstinence'. To prevent psychotic relapses, the first year of treatment targets the unique characteristics of such individuals, focusing on building adherence to medication regimens, abstinence from substance use and insight into schizophrenia. The model provides supportive, cognitive-behavioural, behavioural and motivational therapies, skills building and psychoeducation. At the end of a 12-month treatment trial involving 68 individuals with first-episode schizophrenia or schizoaffective disorder, 30 of whom were using cannabis on diagnosis, nearly half of the cannabis users had stopped using and two were using less frequently. The remainder of the group continued frequent use (Miller 2005).

\section{Literature reviews}

\section{Non-psychotic populations}

A Cochrane review of psychotherapeutic interventions in non-psychotic cannabis misusers analysed six trials involving 1297 people (Denis 2006). Both group and individual sessions of cognitive-behavioural therapy showed efficacy for the treatment of cannabis dependence and associated problems, but the effect was greater with individual sessions. Two studies suggested that adding voucher-based incentives to effective psychotherapeutic interventions may enhance treatment. Abstinence rates were relatively small overall, but favoured nine or more sessions of cognitive-behavioural therapy. The reviewers reported that the included studies were too heterogeneous to allow the drawing of clear conclusions. However, the low abstinence rate indicated that cannabis dependence is not easily treated by psychotherapies in out-patient settings.

\section{Populations with psychotic disorders}

The most recent comprehensive review of psychosocial interventions for people with co-occurring substance use disorder and severe mental illness assessed 45 controlled studies (Drake 2008). Consistent positive effects on substance misuse were found with three types of integrated intervention: group counselling, contingency management and long-term residential treatment. Group counselling appears to be especially beneficial for educational, skills building and peer support purposes. Contingency management interventions mainly focus on substance use and appear promising, but further validation is required. Long-term residential treatment appears to be the most effective, especially for those who have failed other out-patient interventions.

Interventions other than group counselling, contingency management and long-term residential treatment had no significant effect on substance use outcomes, but often led to improvements in other areas of adjustment. The reviewers emphasised the limitations of the studies in their review, mainly lack of standardisation and diversity of chosen outcomes. They called for further experimental research using interventions that are standardised in terms of programme and duration of treatment, outcome, fidelity and adherence measures, staffing patterns, training approaches and other critical parameters. 


\section{Summary}

Most studies and reviews of psychosocial interventions for co-occurring severe mental illness and substance misuse indicate that treatment response is more likely to be a short-term reduction in use, rather than short- or long-term abstinence. It can therefore be said that cannabis dependence cannot easily be treated by psychological interventions and that maintaining non-use is particularly difficult. However, most patients will benefit even from reducing their cannabis consumption.

Only a limited number of studies targeted inpatients, even though substance use may continue in acute care settings, exacerbating treatment and management problems. I have noticed that some patients, especially at the beginning of their admission, can admit to the deleterious effect of their substance use. Perhaps it is still fresh in their memory that it was cannabis that made them suspicious and led to their admission to hospital. If a valid treatment model were available, this level of motivation might be harnessed to encourage reduction or cessation. Clearly, more studies examining effective treatment models for inpatients are also needed.

This section is summarised in Key points 2 .

\section{Pharmacotherapy}

Pharmacotherapy for co-occurring severe mental illness and substance misuse is a recently developing area. It involves study of the pharmacokinetics both of psychotropic medications and substances of misuse and of the interactions between them. It has been suggested that individuals with psychotic disorders may use substances to 'self-treat' the negative symptoms or to reduce the side-effects of antipsychotics. Even though the self-medication hypothesis is disputed, patients have reported that cannabis helps them relax, become more energised and sociable, and that it reduces extrapyramidal

\section{KEY POINTS 2 Psychological interventions}

- Established views that people with severe mental illness and substance use cannot benefit from psychological interventions must be challenged

- Despite methodological limitations, current research studies show consistent positive outcomes following several types of psychological intervention

- There is some evidence, based on a few randomised controlled trials on out-patient groups, that cognitive-behavioural therapy, motivational intervention and a hybrid of other combined therapies can be superior to routine care

- Most patients respond to interventions by reducing their cannabis use, rather than stopping use altogether

- Long-term effects of interventions are not yet encouraging

- There is a great need for more standardised research and well-resourced implementation

- Outcome measures need to be developed and generalised beyond use of substances side-effects (Dixon 1991; Schofield 2006). Hence, targeting the treatment of negative and affective symptoms of schizophrenia or bipolar disorder may have beneficial effects on reducing the drive or the need to use substances. However, in a recent longitudinal study involving patients with firstepisode schizophrenia, persistence with substance use was associated with more severe positive and depressive symptoms, but not with negative symptoms, and the latter were noted as a potential target for specific treatment (Harrison 2007).

\section{Reducing the drive to take substances}

An ideal medicine for an individual with severe mental illness who is using cannabis should reduce not only negative, positive and affective symptoms but also the drive to take stimulants. Typical antipsychotics are thought to worsen substance use, mostly because of their unpleasant side-effects and competing metabolism (McEvoy 1995). However, a recent comprehensive review has revealed a growing body of evidence that some atypicals may have benefits over the typicals in terms of reducing substance misuse in people with schizophrenia (Green 2008).

\section{Clozapine}

Clozapine is the most studied of the atypical antipsychotics in patients with dual diagnosis, and the evidence so far points towards its beneficial effects, especially in alleviating negative and depressive symptoms. A review of substance misuse by people with treatment-resistant schizophrenia revealed that clozapine produced similar improvements in symptoms and psychosocial functioning levels of both users and non-users, and that a history of substance misuse did not appear to have a negative influence on response to clozapine (Buckley 1994). In a prospective study involving alcohol- and cannabis-using patients with schizophrenia and schizoaffective disorder, those receiving clozapine had significantly better remission rates for substance use than those on typical antipsychotics (Drake 2000). A 10-year follow-up report from the same group showed that individuals taking clozapine were less likely to relapse into substance use over the subsequent year than those treated with other antipsychotics (Brunette 2006b). In a retrospective survey of 58 clozapine clinic patients with schizophrenia or schizoaffective disorder and substance use, a significant reduction in cannabis, alcohol and cocaine use was recorded in those who continued to take clozapine, compared with those who discontinued it (Zimmet 2000). The majority of the patients who carried on taking clozapine also achieved abstinence over the period of the 
survey, as well as showing significant clinical improvements. However, the authors did not use any measurement scales to study the changes in substance use. They emphasised the need for prospective, controlled studies to shed further light on the effects of clozapine on substance use in this population.

It has been hypothesised that clozapine's positive effect in the pharmacological treatment of schizophrenia and co-occurring substance misuse may be related to its ability to decrease brain reward circuit dysfunction (Green 2006). It has also been suggested that the weekly professional contact that clozapine patients experience helps sustain a stronger therapeutic alliance (Buckley, 1998). However, in the absence of any randomised studies, claims that clozapine has a beneficial effect on substance misuse in severe metal illness remain preliminary.

\section{Clozapine v. risperidone}

A retrospective study comparing clozapine with risperidone involving 41 individuals with schizophrenia and comorbid alcohol and/or cannabis misuse found that patients treated with clozapine were more likely to abstain from substance use than those treated with risperidone (Green 2003).

\section{Other atypicals}

Preliminary reports are available on the use of all other atypical antipsychotics in people with dual diagnosis except for ziprasidone. However, most of these studies are retrospective, non-randomised and include only small numbers of participants. Furthermore, the study measures vary widely, making comparisons impossible.

Quetiapine has raised interest because it shares crucial pharmacological properties with clozapine. In a small study, 8 in-patients treated with quetiapine for comorbid substance dependence and non-psychotic anxiety remained abstinent during the 28-day trial and showed reduced anxiety (Sattar 2004). A 12-week, open-label uncontrolled trial involving 24 people with schizophrenia and substance use recorded significant improvements in substance misuse, psychiatric symptoms, extrapyramidal symptoms and cognition during quetiapine therapy (Potvin 2006).

Olanzapine and haloperidol were compared in the treatment of 262 individuals with first-episode schizophrenia-related psychosis, 97 (37\%) of whom had a lifetime diagnosis of substance use disorder (Green 2004). The 12-week response data indicated that $27 \%$ of the participants with substance use disorder responded to either medication, compared with 35\% of those without.

Individuals with alcohol use disorder were less likely to respond to olanzapine than those without. The authors conclude that the use of substances or alcohol in first-episode psychosis may negatively affect the response to both typical and atypical antipsychotics.

\section{Caveat: smoking cessation and drug plasma levels}

Blood plasma levels of antipsychotics such as clozapine and olanzapine are lower in smokers than in non-smokers, mainly because of induction of cytochrome P450 enzymes 1A2 (CYP1A2) by constituents of tobacco smoke (Faber 2004). When the patient stops smoking, plasma levels of antipsychotics may increase, leading to adverse drug effects, including antipsychotic intoxication. Reporting this in two patients, Zullino and colleagues (2002) warn that antipsychotic plasma levels should be monitored regularly in patients who smoke tobacco or cannabis, to adjust the dose according to a reduction or increase in smoking.

This section is summarised in Key points 3 .

\section{Conclusions}

Cannabis use clearly has serious implications for severe mental illness. The problem is further complicated by poor assessment, lack of successful educational campaigns and, most significantly, lack of successful models of intervention. However, encouraging research is emerging to inform management approaches. Patients should receive the same message from all clinicians, that the treatment of both their mental health problems and cannabis or substance use is equally important.

There have been positive results from the hybrid use of various psychological interventions for substance misuse in severe mental illness, even though these need fine tuning. Pharmacotherapy for this group of patients is also in its early stages, but there

\section{KEY POINTS 3 Psychopharmacological interventions}

- Typical antipsychotics for substance use are not beneficial and may even increase use

- The available data shows consistent, positive effect of clozapine in this population. However, more studies with better methodology are required to reach firmer conclusions

- Quetiapine appears to have some beneficia effect, but this should be regarded with caution because the data were derived from two small prospective studies, without controls
- Some small randomised studies have reported beneficial effect with olanzapine and risperidone, but other studies have found no such effect

- More randomised prospective studies with larger numbers of patients and use of appropriate measurement scales are required

- Cannabis smoking cessation may lead to clozapine or olanzapine intoxication; drug plasma levels should therefore be monitored and doses adjusted according to the reduction or increase in tobacco and cannabis use 


\begin{tabular}{lllll}
\multicolumn{5}{l}{ MCQ answers } \\
$\begin{array}{lllll}1 & 2 & 3 & 4 & 5 \\
\text { af } & \text { at } & \text { af } & \text { af } & \text { at } \\
\text { bf } & \text { bf } & \text { bf } & \text { bt } & \text { bf } \\
\text { cf } & \text { cf } & \text { cf } & \text { cf } & \text { cf } \\
\text { dt } & \text { df } & \text { df } & \text { df } & \text { df } \\
\text { ef } & \text { ef } & \text { et } & \text { ef } & \text { ef }\end{array}$
\end{tabular}

is overall agreement that atypical antipsychotics may prove to be better than the typicals. However, it must be remembered that substance misuse behaviour cannot be explained by pharmacokinetics alone. Adding contingency management, psychoeducation and social skills training may enhance the efficacy of pharmacotherapy. Furthermore, most agree that programmes that coordinate pharmacotherapy and psychological interventions are more likely to have beneficial treatment outcomes. It is also important that some meaningful activity replaces the drug-misusing behaviour, as maintaining cannabis or substance use cessation is often the most challenging aspect. More standardised, prospective, randomised and substance-specific research is required to establish an efficient model to help people with severe mental illness to reduce or stop their cannabis use and maintain abstinence.

\section{References}

Barrowclough C, Haddock C, Tarrier N, et al (2001) Randomized controlled trial of motivational interviewing, cognitive behavior therapy, and family intervention for patients with comorbid schizophrenia and substance use disorders. American Journal of Psychiatry; 158: 1706-13.

Brunette MF, Mueser KT (2006a) Psychosocial interventions for the long-term management of patients with severe mental illness and co-occurring substance use disorder. Journal of Clinical Psychiatry; 67 (suppl 7): 10-7.

Brunette MF, Drake RE, Xie H, et al (2006b) Clozapine use and relapses of substance use disorder among patients with co-occurring schizophrenia and substance use disorders. Schizophrenia Bulletin; 32: 637-43.

Buckley PF, Thompson P, Way L, et al (1994) Substance abuse among patients with treatment-resistant schizophrenia: characteristics and implications for clozapine therapy. American Journal of Psychiatry; 151: 385-9.

Buckley PF (1998) Substance abuse in schizophrenia. A review. Journal of Clinical Psychiatry; 3 (suppl 59): 26-30.

Burnam MA, Morton SC, McGlynn EA, et al (1995) An experimental evaluation of residential and nonresidential treatment for dually diagnosed homeless adults. Journal of Addictive Diseases; 14: 111-34

Cleary M, Hunt GE, Matheson SL, et al (2008) Psychosocial interventions for people with both severe mental illness and substance misuse. Cochrane Database of Systematic Reviews; issue 4.

Denis C, Lavie E, Fatséas M, et al (2006) Psychotherapeutic interventions for cannabis abuse and/or dependence in outpatient settings. Cochrane Database of Systematic Reviews; issue 3

Dixon L, Haas G, Weiden PJ, et al (1991) Drug abuse in schizophrenic patients: clinical correlates and reasons for use. American Journal of Psychiatry; 148: 224-30.

Drake RE, Xie H, McHugo GJ, et al (2000) The effects of clozapine on alcohol and drug use disorders among patients with schizophrenia. Schizophrenia Bulletin; 26: 441-9.

Drake RE, O'Neal EL, Wallach MA (2008) A systematic review of psychosocial research on psychosocial interventions for people with co-occurring severe mental and substance use disorders. Journal of Substance Abuse Treatment; 34: 123-38.

Faber MS, Fuhr U (2004) Time response of cytochrome P450 1A2 activity on cessation of heavy smoking. Clinical Pharmacology and Therapeutics; 76 : $178-84$.

Green Al (2006) Treatment of schizophrenia and co-morbid substance abuse: pharmacologic approaches. Journal of Clinical Psychiatry; 67 (suppl 7): $31-5$.
Green Al, Burgess ES, Dawson R, et al (2003) Alcohol and cannabis use in schizophrenia. Effects of clozapine vs. Risperidone. Schizophrenia Research; 60: $81-5$.

Green Al, Tohen MF, Hamer RM, et al (2004) First episode schizophreniarelated psychosis and substance use disorders. Acute response to olanzapine and haloperidol. Schizophrenia Research; 66: 125-35.

Green Al, Noordsy DL, Brunette MF, et al (2008) Substance abuse and schizophrenia: pharmacotherapeutic intervention. Journal of Substance Abuse Treatment; 34: 61-71

Haddock G, Barrowclough C, Tarrier N, et al (2003) Cognitive-behavioural therapy and motivational intervention for schizophrenia and substance misuse: 18-month outcomes of a randomised controlled trial. British Journal of Psychiatry; 183: 418-26.

Harrison I, Joyce EM, Mutsatsa SH, et al (2007) Naturalistic follow-up of comorbid substance use in schizophrenia: the West London first-episode study. Psychological Medicine; 29: 1-10.

James W, Preston NJ, Koh G, et al (2004) A group intervention which assists patients with dual diagnosis reduce their drug use: a randomized controlled trial. Psychological Medicine; 34: 983-90.

Krabbendam L, Arts B, van Os J, et al (2005) Cognitive functioning in patients with schizophrenia and bipolar disorder. A quantitative review. Schizophrenia Research: 80: 137-49.

Martino S, Carroll K, Kostas D, et al (2002) Dual diagnosis motivational interviewing. A modification of Motivational Interviewing for substanceabusing patients with psychotic disorders. Journal of Substance Abuse Treatment; 23: 297-308.

McCambridge J, Strang J (2004) The efficacy of single-session motivational interviewing in reducing drug consumption and perceptions of drug-related risk and harm among young people: results from a multi-site cluster randomized trial. Addiction; 99: 39-52.

McEvoy JP, Freudenreich 0, Levin ED, et al (1995) Haloperidol increases smoking in patients with schizophrenia. Psychopharmacology; 119: 124-6.

Miller WR, Rollnick S (2002) Motivational Interviewing: Preparing People for Change. Guilford Press.

Miller R, Caponi JM, Sevy S, et al (2005) The Insight-Adherence-Abstinence triad: an integrated treatment focus for cannabis-using first-episode schizophrenia patients. Bulletin of the Menninger Clinic; 69: 220-36.

Potvin S, Stip E, Lipp 0, et al (2006) Quetiapine in patients with comorbid schizophrenia-spectrum and substance use disorders: an open-label trial. Current Medical Research and Opinion; 22: 1277-85.

Prochaska J0, DiClemente CC (1992) Stages of change in modification of problem behaviours. Progress in Behavior Modification; 28: 183-218.

Sattar SP, Bhatai SC, Petty F (2004) Potential benefits of quetiapine in the treatment of substance dependence disorders. Journal of Psychiatry and Neurosciences; 29: 452-7.

Schofield D, Tennant C, Nash L, et al (2006) Reasons for cannabis use in psychosis. Australian and New Zealand Journal of Psychiatry; 40: 570-4.

Stroup S, Appelbaum P, Swartz MS, et al (2005) Decision-making capacity for research participation among individuals in the CATIE schizophrenia trial. Schizophrenia Research; 80: 1-8.

Weaver T, Renton A, Stimson G, et al (1999) Severe mental illness and substance misuse. Research is needed to underpin policy and services of patients with comorbidity. BMJ; 318: 137-8.

Ziedonis DM, Trudeau K (1997) Motivation to quit using substances among individuals with schizophrenia. Implications for a motivation-based treatment model. Schizophrenia Bulletin; 23: 229-38.

Zimmet SV, Strous RD, Burgess ES, et al (2000) Effects of clozapine on substance use in patients with schizophrenia and schizoaffective disorder. A retrospective survey. Journal of Clinical Psychopharmacology; 20: 94-8.

Zullino DF, Delessert D, Eap CB, et al (2002) Tobacco and cannabis smoking cessation can lead to intoxication with clozapine and olanzapine. International Clinical Psychopharmacology; 17: 141-3. 


\section{MCOs}

1 Regarding psychological interventions for cannabis use in people with severe mental Illness:

a motivational interviewing is sufficient to help the person

b cognitive-behavioural therapy is not suitable

c psychological interventions have shown long-term benefits

d most patients respond to interventions by reducing their cannabis use, rather than stopping use altogether

e they have no significant effect over standard care.

\section{Regarding pharmacological treatment of cannabis} use in people with severe mental illness:

a typical antipsychotics may worsen cannabis use

b all atypical antipsychotics have been shown to reduce cannabis use

c clozapine is not superior to any other antipsychotic

d there is good evidence that most atypicals can reduce cannabis use

e cannabis smoking cessation reduces the possibility of intoxication with clozapine or olanzapine.
3 For people with severe mental illness who use cannabis:

a combining various psychological intervention models is always beneficial

b programmes that coordinate pharmacotherapy and psychological interventions are less likely to have beneficial treatment outcomes

c self-medication theory is valid

d early use of cannabis has been shown to be associated with development of a psychotic illness

e assessment of cannabis use should include its effect on physical health.

4 Regarding people with severe mental illness:

a for those who also use substances integrated treatment is superior to other models

b models for dual diagnosis intervention require development and testing before widespread implementation

c most such individuals cannot benefit from psychological interventions, owing to their cognitive deficits d reducing cannabis use is of no benefit: complete cessation is necessary

e psychoeducation regarding cannabis use is not effective.

5 The following statements are true:

a motivational interviewing is informed by the following stages: pre-contemplation, contemplation, preparation, action, maintenance

b knowing why a patient uses cannabis is not necessarily important in designing a treatment plan

c following treatment for substance use, establishing the patient's level of substance consumption is a sufficient measure of outcome

d during the maintenance phase of the treatment, regular follow-up is sufficient to ensure success

e substance use research need not be substance specific, as all substances have similar negative impact on the patient's course of illness. 